\title{
Pilot study on effectiveness of a virtual game training on executive functions
}

\author{
$1^{\text {st }}$ Irene Alice Chicchi Giglioli \\ Instituto de Investigación e Innovación \\ en Bioingeniería (i3B) \\ Universitat Politécnica de Valencia \\ Valencia, Spain \\ alicechicchi@i3b.upv.es \\ $4^{\text {th }}$ Javier Marín-Morales \\ Instituto de Investigación e Innovación \\ en Bioingeniería (i3B) \\ Universitat Politécnica de Valencia \\ Valencia, Spain \\ jamarmo@i3b.upv.es
}

\author{
$2^{\text {nd }}$ Sara Mussoni \\ Department of Psychology \\ Catholic University of the Sacred Heart \\ Millan, Italy \\ sara.mussoni01@icatt.it
$5^{\text {th }}$ Giuseppe Riva
Department of Psychology
Catholic University of the Sacred Heart
Millan, Italy
giuseppe.riva@unicatt.it

\author{
$3^{\text {rd }}$ Pietro Cipresso \\ Department of Psychology \\ Catholic University of the Sacred Heart \\ Millan, Italy \\ Pietro.Cipresso@unicatt.it \\ $6^{\text {th }}$ Mariano Alcañiz \\ Instituto de Investigación e Innovación \\ en Bioingeniería ( $i 3 B$ ) \\ Universitat Politécnica de Valencia \\ Valencia, Spain \\ malcaniz@i3b.upv.es
}

\begin{abstract}
Attention, control inhibition, and visual-spatial working memory represent the three basic sets of cognitive processes involving on executive functions (EF). Basic EF are relevant abilities in daily life that allow to control and monitor adapted behaviors in order to achieve specific goals. In the educational field, EF are related to academic achievement, social functioning, as well as the inhibition of maladaptive behaviors. Their impairment often leads to an incapacity to perform multiple and simultaneous mental activities, as well as to plan and monitor learning. The main aim of cognitive neuropsychology intervention is to identify effective methods that allow transferring trained strategies and abilities to daily life. Accordingly, virtual reality games (VRG) are showing ecological validity effectiveness in EF training. In this framework, the aim of this study was to examine the effectiveness of a VRG cooking-based for improving basic EF processing. 31 healthy subjects $(M=24.3 ; \quad S D=2.51)$ participated to 3 training sessions of 25 minutes each. Each session involved 6 VRG characterized by different levels of difficulties. Three traditional measures were administered to participants pre- and post- VRG: The Corsi test for assessing visual-spatial working memory, the Dual-task, and the Flanker task for attention and inhibition control respectively. The results reported a significant improvement of the three EF abilities after training, showing the potential effectiveness of a VRG along with the traditional measures. Future studies on students with learning disabilities are needed to compare performance and effectiveness.
\end{abstract}

Keywords- Executive functions, virtual reality, educational training

\section{INTRODUCTION}

Attention, control inhibition, and visual-spatial working memory represent the three basic sets of cognitive processes involving on executive functions (EF) [1]. Executive functioning is a multidimensional paradigm that contribute to the formation, preservation and shifting of mental sets [2]. Basic EF are relevant learning abilities in daily life that allow to acquire, control and monitor adapted behaviours in order to achieve specific goals [3]. In the educational field, EF are related to academic achievement, social functioning, as well as the inhibition of maladaptive behaviours [4]. Specifically, attention is the first cognitive ability in the learning process of selectively concentrating on one or some discrete stimuli, disregarding other perceivable stimuli. Through attention abilities, learners can prioritize information to retain in memory, in order to apply the acquired knowledge in various learning and daily life contexts. Related to attention in learning, visual-spatial working memory allow learners to visualize and retain information, such as numbers, sequences and spatial relations among objects for established time. Finally, inhibitory control allows to remove inappropriate memories or information, as well as to resist interference from distracting stimuli in order to make a decision or solve a problem $[1,2]$.

Their impairment of the EF often leads to an incapacity to perform multiple and simultaneous mental activities, as well as to plan and monitor learning. The main aim of cognitive neuropsychology intervention and training is to identify effective methods that allow transferring trained strategies and abilities to daily life [4]. In recent years, there has been a relevant interest in the development of EF training, mainly focused on children and older adults $[5,6]$. EF training programs for children are considered a useful approach to reduce neurocognitive learning disparities among children, increasing academic achievements and promoting positive development outcomes. With regard to older adults, EF training programs are showing to be able to reduce and curb cognitive impairments and frailties. Traditional EF programs differ in duration (short vs. longterm), setting (individual vs group training program), and methods. Regarding methods, several types of training faceto-face have been developed and tested, showing, on the one hand, efficacy both in children and older adults, and on the other hand, limitations in term of real-performance. Indeed, several research are showing that patients who show poor EF' abilities in their daily life activities are performing within normal limits of neuropsychological training on EF and vice-versa. This incongruence could be due to the fact that neuropsychological training is too abstract and does not reflect the complexity and dynamicity of real-life activities and situations. Moreover, traditional trainings are criticized because of each training attempts to foster a specific EF when the functioning is characterized by multidimensionality and simultaneousness.

In order to overcome these limitations, the creation of more ecologically valid neuropsychological trainings are showing the enhancement of results to be generalized to situations and activities of daily living. Accordingly, a new approach has recently emerged, potentially providing a higher ecological validity in functional cognitive abilities training than standardized approach: the use of virtual reality games (VRG). These games consist of interactive 
synthetic simulations that allow users to perform in environments similar to real ones [7]. Educational VRG can provide experiential and active learning during engaging activities, stimuli control and degree of task difficulty, as well as the possibility of objectively measuring user behaviours [8]. VRG are showing to be suitable tools for training, rehabilitation, and empowerment of EF regarding daily life activities and learning [9-12]. Indeed, studies on educational VRG have showed to be able to motivate learners, developing positive attitudes, patience and problem-solving skills. Therefore, in the educational field, VRG represent an encouraging area for improving learning abilities and disabilities. A recent review of Alabdulakareem and Jamjoom [9] showed the effectiveness of VRG to improve EF in attention-deficit/hyperactivity disorder (ADHD). For example, Dovis et al. [13] developed a game called "BrainGame Brian", consisting of 25 sessions of 40-50 minutes each during 5 weeks in which they determined the short and long term effects of the training on multiple EF, including working memory, control inhibition, and cognitive flexibility. Each session consisted of three tasks related to the three EF. "Mathloons" and "Space Motif" are other two games developed for the improvement of EF [14]. Mathloons is a mathematical game to train math operations and consisted of 8-11 sessions, each lasting 30 minutes for 5 weeks and "Space Motif" aimed to train ordering, sorting, pattern-matching and spatiotemporal skills. The results on both games showed an improvement in learners EF abilities. Furthermore, another relevant outcome on using VRG with respect to traditional methods, concerns the duration of training that despite the time duration is short, the results are proving positive and long term effects. Similar results have been found also in older people using mainly virtual supermarket or shopping mall or virtual city as a virtual context [6]. For example, Riva et al. developed a virtual mall training in which patients should move in the supermarket, selecting established products on the shelves following precise rules. The training consisted of 10 different tasks increasing in difficulty and the preliminary results showed positive improvements after training in cognitive frail patients. Despite the positive results, the main virtual applications have been developed for non-immersive or semi-immersive virtual systems, such as desktop or Cave Automatic Virtual Environments, and less frequently for immersive systems, like a head mounted displays [9].

Starting from these premises, we developed a narrative and immersive VRG for the empowerment of multiple and simultaneous EF. Precisely, the goal of the present pre-post pilot study was to investigate the effectiveness of $E F$ training using a VRG, and we assumed a strengthening of the EF after the training through an improvement in the scores of the tests selected to evaluate multiple and simultaneously functions. If these hypotheses were verified, it would initially demonstrate the effectiveness of the training carried out in virtual reality.

\section{MATERIALS AND METHOD}

\section{A. Participants}

The study sample included 31 individuals (mean \pm SD age was $24.3 \pm 2.51$ years; 12 were males and 19 females). Sample size $(\mathrm{N}=31)$ was predetermined by a power analysis $\left(\mathrm{G}^{*}\right.$ Power 3.1; Faul et al. 2007) for $t$-test, given $\mathrm{f}=$ 0.25 , power $=0.8, \alpha=0.05$. The participants were recruited within the Catholic University of Milan, through notices on Facebook and LinkedIn. Participation in the study was voluntary and no form of monetary contribution was provided to participants. The ethical committee of the Catholic University of Milan approved the study and all participants signed an informed consent prior to enrollment and study participation.

\section{B. Neuropsychological instruments to measure outcomes}

- Corsi test. It is an assessment tool designed to examine visual-spatial working memory [16]. The test consists of nine numbered cubes of $32 \times 25$ that the examiner touches following standard sequences of increasing length (from two to ten cubes) and the subject has to repeat the sequence in the same order of presentation. After executing 3 correct sequences, the examiner moves on to longer sequences. The number of cubes of the major sequence correctly reproduced by the subject 3 times represents the visual-spatial span.

- Dual-task. It is an evaluation designed to examine attention on two tasks simultaneously [17]. It is composed by 4 tests: a) the first, named "Digit span determination" consists of the experimenter's reading of a sequence of numbers of increasing length (from two to ten numbers) that the participant will have to repeat in the same order. Once the subject repeats 5 numbers correctly, he moves on to the next sequence. When the participant misses more than 5 sequences, the Digit Span (n) will be represented by the major sequence correctly repeated by the subject 5 times; b) in the second test "Learning lists of numbers in single task" a sequence of numbers is repeated based on the span obtained in the previous test and the participant must repeat the lists of digits in the same order of presentation. For each list, the number of correctly repeated digits in the sequence is calculated $(\mathrm{P})$ and the number of digits repeated correctly with respect to the span (P / $\mathrm{n})$. The indices that are calculated are: ns, Ns and ps. The first index (ns) is given by the sum of the numbers correctly repeated with respect to the span in each sequence divided by the total number of strings $(\mathrm{ns}=(\mathrm{P} / \mathrm{n}+\mathrm{P} / \mathrm{n}+\ldots) / 15)$. Ns represents the number of strings read in a minute and a half of time on the total number of strings present. The last index (ps) is given by the ratio between the first two indices ( $\mathrm{ps}=\mathrm{ns} / \mathrm{Ns})$; $\mathrm{c}$ ) In the third test called "Tracking in Single Task" the subject is given an A3 sheet with a set of dots that form a path and the subject, in 1 and a half minutes, has the task of tracing a road, starting from the beginning of path, passing through the dots without removing the pen from the sheet. The score (ts) that is calculated is given only by the number of white circles crossed out, excluding the dots not drawn on the course, on the total of 330 circles present on the sheet; d) In the fourth and last test, called "Span and Tracking in Dual Task", the second and third tests are repeated simultaneously. The subject has 1 minute and a half to perform the two tasks simultaneously, that is, to trace the path through the dots while repeating the list of numbers read by the examiner. In this last test the same indices are calculated and, in addition, the 
difference between the score obtained in single (ps, ts) and the score obtained in dual (pd, td) is calculated both as regards the learning test of lists of digits $(\mathrm{pm}=\mathrm{ps}-\mathrm{pd})$ and with respect to the tracking test $(\mathrm{pt}=(\mathrm{ts}-\mathrm{td}) / \mathrm{ts})$.

- Flanker task. It is an assessment designed to examine control inhibition [18]. The adopted version was created on Neuropsydia, a Python module through which it is possible to carry out tests and cognitive tasks. The subject completed the computer test with a total duration of 3 minutes in which 150 trials followed one another. A central arrow represents the target stimulus whose sides (right and left) have other arrows in the same or different direction. The subject's task is to focus attention on the direction of the arrow in the centre (right or left) and, consequently, press on the computer keyboard the arrow pointing to the right or to the left according to the direction of the target stimulus. The stimuli were randomized and three conditions were distinguished: congruent, incongruent and neutral. In the congruency condition, the arrows placed at the side have the same direction as the target arrow in the centre. In the condition of incongruity, instead, the direction of the arrows does not correspond to the direction of the target stimulus. Finally, in the condition of neutrality the arrows are turned upwards or downwards not coming into conflict with the target stimulus, whose direction is right or left.

\section{The VRG to provide training}

The VRG environment simulated cooking a meal, which was examined as a valid assessment tool in neuropsychology and no studies have used it as a training tool [19]. For training purpose, 6 tasks have been developed and graded according to difficulty in each of the 3 training sessions Each task lasted three and a half minutes and the difficulty increased according to the number and type of stimuli presented and the presentation time for each (Table 1).Each task has been accompanied by positive visual (e.g. scores obtained), audio and verbal feedbacks, motivational cues and/or suggestions on the best strategies users could use. Each task has been designed according to the specific EF psychological construct and its functioning in daily life activities. The VRG was developed using Unity 5.5.1f1 software, applying C\# programing language using the Visual Studio tool.

\section{TABLE I. CHARACTERISTICS OF EACH TASK AND} DIFFICULTY LEVEL

\begin{tabular}{|c|c|c|}
\hline $\begin{array}{c}\text { Task } \\
\text { Time: } 3.30 \\
\text { minutes }\end{array}$ & Difficulty Level Increase & Executive Functions \\
\hline $\begin{array}{l}\text { Kitchen } \\
\text { Utensils } \\
\text { Search }\end{array}$ & $\begin{array}{ll}- & \text { Number of kitchen } \\
\text { drawers } \\
\text { - } & \text { Type of kitchen } \\
& \text { utensil } \\
\text { - } & \text { Time stimuli } \\
& \text { presentation }\end{array}$ & $\begin{array}{ll}- & \begin{array}{l}\text { Attention } \\
\text { (sustained and }\end{array} \\
\text { divided) } \\
\text { - } & \text { Control } \\
\text { Inhibition }\end{array}$ \\
\hline $\begin{array}{c}\text { Recipe } \\
\text { ingredients }\end{array}$ & $\begin{array}{l}\text { Number of } \\
\text { ingredients to recall } \\
\text { increase }\end{array}$ & $\begin{array}{ll}- & \text { Working } \\
& \text { Memory } \\
\text { - } & \text { Attention }\end{array}$ \\
\hline $\begin{array}{l}\text { Cook in the } \\
\text { burners }\end{array}$ & $\begin{array}{ll}- & \text { Time stimuli } \\
& \text { presentation } \\
- & \text { Speed of stimuli } \\
& \text { presentation }\end{array}$ & $\begin{array}{ll}- & \text { Attention } \\
- & \text { Working } \\
& \text { Memory }\end{array}$ \\
\hline
\end{tabular}

\begin{tabular}{|c|cl|ll|}
\hline $\begin{array}{c}\text { Burner } \\
\text { Adjust }\end{array}$ & - & $\begin{array}{l}\text { Speed of stimuli } \\
\text { presentation } \\
\text { Time of stimuli } \\
\text { presentation }\end{array}$ & - & $\begin{array}{l}\text { Sustained } \\
\text { attention } \\
\text { Divided } \\
\text { attention }\end{array}$ \\
& - & $-\begin{array}{l}\text { Control } \\
\text { Inhibition }\end{array}$ \\
\hline $\begin{array}{c}\text { Hidden } \\
\text { Ingredients } \\
\text { search }\end{array}$ & - & $\begin{array}{l}\text { Speed of stimuli } \\
\text { presentation } \\
\text { Number of stimuli } \\
\text { presented }\end{array}$ & - & $\begin{array}{l}\text { Attention } \\
\text { Control } \\
\text { Inhibition }\end{array}$ \\
\hline $\begin{array}{c}\text { Cooking a } \\
\text { Cake }\end{array}$ & - & $\begin{array}{l}\text { Speed of stimuli } \\
\text { presentation } \\
\text { Number of stimuli } \\
\text { presented }\end{array}$ & - & $\begin{array}{l}\text { Attention } \\
\text { Working } \\
\text { Memory } \\
\text { Control }\end{array}$ \\
& - & - & \begin{tabular}{l} 
Inhibition \\
\hline
\end{tabular} \\
\hline
\end{tabular}

\section{Experimental protocol}

The study followed a pre-post design and the protocol included three phases:

1. Pre-training assessment: standardized paper and pencil tests designed to measure visual-spatial working memory, attention and control inhibition, were administered to participants before the training sessions.

2. Training sessions: all participants performed 3 sessions of 30 minutes each. Each session involved 6 VRG characterized by different levels of difficulties. The sessions were every other day and the total training duration did not exceed two weeks. The VRG training was experienced used a head mounted display (HTC VIVE/Pro) and the first part of the first session was dedicated to familiarize with the controllers and understanding which commands to use to carry out specific movements or actions (e.g. catching, moving, touching, etc.).

3. Post-training assessment: the three standardized paper and pencil tests designed to measure attention, control inhibition, and visual-spatial working memory were administered to participants.

\section{E. Data analysis}

Data were analysed using Jamovi 1.0.4.0. Pre- and postdifferences in performance on neuropsychological tests were evaluated using t-test for paired samples, due to the normality of the data ( $p>0.05$ from the Shapiro-Wilk test with null hypothesis of having a Gaussian sample).

\section{RESULTS}

\section{A. Descriptive analysis}

The sample of study participants was made up of 31 subjects, of which 12 were male $(38.7 \%)$ and 19 were female $(61.3 \%)$ (Table 2). Participants had an average age of $24.3(\mathrm{SD}=2.51)$ (Table 3$)$ and the $74 \%$ were students and $100 \%$ unmarried (Table 4 ).

TABLE II. GENDER DISTRIBUTION OF THE SAMPLE

\begin{tabular}{|l|c|c|}
\hline & Frecuency & $\%$ \\
\hline Male & 12 & $38.7 \%$ \\
\hline Female & 19 & $61.3 \%$ \\
\hline Tot. & 31 & $100.0 \%$ \\
\hline
\end{tabular}


TABLE III. SAMPLE AGE

\begin{tabular}{|c|c|c|c|c|c|}
\hline & $\boldsymbol{N}$ & Min. & Max. & $\boldsymbol{M}$ & SD \\
\hline Age & 31 & 21 & 31 & 24.3 & 2.51 \\
\hline
\end{tabular}

TABLE IV. SOCIODEMOGRAPHIC VARIABLES

\begin{tabular}{|c|c|c|}
\hline \multicolumn{3}{|c|}{ Marital Status } \\
\hline Sociodemographics & Frecuency & $\%$ \\
\hline Unmarried & 31 & $100.0 \%$ \\
\hline \multicolumn{3}{|c|}{ Student/Worker } \\
\hline Student & 23 & $74.2 \%$ \\
\hline Worker & 8 & $25.8 \%$ \\
\hline High school Degree & 4 & $12.9 \%$ \\
\hline Bachelor Degree & 18 & $58.1 \%$ \\
\hline Master Degree & 9 & $29.0 \%$ \\
\hline \multicolumn{3}{|c|}{ VR use } \\
\hline Yes & 23 & $74.2 \%$ \\
\hline No & 8 & $25.8 \%$ \\
\hline
\end{tabular}

\section{B. Corsi Test}

A t-test was carried out for paired samples to check if there was a significant difference in the visual-spatial span obtained in the Corsi Test before and after training. Table 5 shows the main significant difference between the visualspatial span averages before and after training.

\section{Dual Task}

The t-test for paired samples revealed a significant difference in relation to both the "Digit span determination" test and the "Tracking" task in the single and dual condition. As regards the "Learning of lists of numbers" test, no significant results have emerged (Table 6).

\section{Flanker Test}

The t-test results showed a significant difference in reaction times in the congruence condition (RT Cong) and in the neutrality condition (RT Neu) between the averages of these indices before and after training (Table 7). No significant differences have emerged in reaction times in the incongruence condition. With regard to the correct answers and the number of errors committed by the participants in the three conditions (congruent, incongruent and neutral), no significant differences emerged between the averages obtained before and after the training.

TABLE V. CORSI T-TEST RESULTS -PRE AND -POST VRG TRAINING

\begin{tabular}{|c|c|c|c|c|c|}
\hline & Mean & $\boldsymbol{S D}$ & $\boldsymbol{t}$ & $\boldsymbol{d} \boldsymbol{f}$ & $\boldsymbol{p}$ \\
\hline Span_Pre & 5.90 & 0.746 & - & \multirow{2}{*}{30.0} & \multirow{2}{*}{0.005} \\
\cline { 1 - 4 } Span_Post & 6.32 & 0.653 & 3.05 & & \\
\hline
\end{tabular}

TABLE VI. DUAL TASK T-TEST RESULTS -PRE AND POST VRG TRAINING

\begin{tabular}{|c|c|c|c|c|c|}
\hline Test & Mean & $S D$ & $t$ & $d f$ & $p$ \\
\hline Digit Span -Pre1 & 6.290 & 1.216 & \multirow{2}{*}{-3.321} & \multirow{2}{*}{30.0} & \multirow{2}{*}{0.002} \\
\hline Digit Span -Post & 6.774 & 1.086 & & & \\
\hline Tracking ts -Pre & 0.663 & 0.201 & \multirow{2}{*}{-6.905} & \multirow{2}{*}{30.0} & \multirow{2}{*}{$<.001$} \\
\hline Tracking ts - Post & 0.853 & 0.242 & & & \\
\hline Tracking td -Pre & 0.650 & 0.191 & \multirow{2}{*}{-6.283} & \multirow{2}{*}{30.0} & \multirow{2}{*}{$<.001$} \\
\hline Tracking td -Post & 0.777 & 0.229 & & & \\
\hline Tracking pt -Pre & 0.002 & 0.176 & \multirow{2}{*}{-2.602} & \multirow{2}{*}{30.0} & \multirow{2}{*}{0.014} \\
\hline Tracking pt -Post & 0.083 & 0.111 & & & \\
\hline
\end{tabular}

TABLE VII. FLANKER TASK T-TEST RESULTS -PRE AND -POST VRG TRAINING

\begin{tabular}{|c|c|c|c|c|c|}
\hline & Mean & $\boldsymbol{S D}$ & $\boldsymbol{t}$ & $\boldsymbol{d} \boldsymbol{f}$ & $\boldsymbol{p}$ \\
\hline RT Cong_Pre & 446 & 56.5 & \multirow{2}{*}{2.07} & \multirow{2}{*}{30.0} & 0.047 \\
\cline { 1 - 3 } RT Cong_Post & 425 & 59.0 & & & \\
\cline { 1 - 4 } RT Neu_Pre & 449 & 57.9 & \multirow{2}{*}{2.24} & \multirow{2}{*}{30.0} & 0.032 \\
\cline { 1 - 4 } RT Neu_Post & 428 & 57.6 & & & \\
\hline
\end{tabular}

\section{DISCUSSION}

The aim of this pilot study was to examine the effectiveness of a multiple and simultaneous EF training using a narrative and immersive VRG. 31 participants took part in a 3-session training consisting mainly of cook a meal tasks within a virtual kitchen. The results on pre-post traditional tests on visual-spatial working memory, attention, and control inhibition showed that VRG could be an effectiveness method to improve EF, confirming our initial hypothesis. The results are coherent with previous works on the effectiveness of EF training using virtual systems [9]. The study presents also limitations in terms of small sample, participants' ages, and lack of a control group; the results must be interpreted with cautious and mainly used as a support for the design of further studies. Furthermore, a relevant aim using VRG for training is to show that the abilities trained within a virtual environment will transfer to real-life activities. Further studies are needed in order to assess performance of these activities as performed in a real kitchen. Finally, taking into consideration also the performance data gathered from the VRG, a future machine learning analysis could accurately predict the relationship between the VRG tasks and the specific EF.

\section{CONCLUSIONS}

On the basis of these preliminary findings, the immersive VRG based on cooking a meal appears to have potential for use as a motivating and effective training tool for multiple and simoultaneous $\mathrm{EF}$, which are related to academic achievement, social functioning, as well as the inhibition of maladaptive behaviours. Furthermore, the VRG allowed repetitive practice of abilities tasks in a safe, realistic, and interactive manner that motivate participants 
to train for multitasking activities similar to those necessary for participation in every day living.

\section{REFERENCES}

[1] Jacques, S., \& Marcovitch, S., Development of executive function across the life span. The handbook of life - span development, 2010.

[2] Diamond, A., Executive functions. Annual review of psychology, 64, 2013, 135-168.

[3] Altemeier, L., Jones, J., Abbott, R. D., \& Berninger, V. W., Executive functions in becoming writing readers and reading writers: Note taking and report writing in third and fifth graders. Developmental neuropsychology, 29(1), 2006, 161-173.

[4] Hofmann, W., Schmeichel, B. J., \& Baddeley, A. D., Executive functions and self-regulation. Trends in cognitive sciences, 16(3), 2012, 174-180.

[5] Watson, S. M., Gable, R. A., \& Morin, L. L., The role of executive functions in classroom instruction of students with learning disabilities. International Journal of School and Cognitive Psychology, 3(167), 2016.

[6] Pedroli, E., Serino, S., Stramba-Badiale, M., \& Riva, G., An innovative virtual reality-based training program for the rehabilitation of cognitive frail patients. In Pervasive Computing Paradigms for Mental Health, Springer, Cham, 2016, 62-66.

[7] F. Mantovani, G. Castelnuovo, The sense of presence in virtual training: enhancing skills acquisition and transfer of knowledge through learning experience in virtual environments, 2003, 167-182.

[8] Parr, J. M., \& Fung, I. Y., A review of the literature on computerassisted learning, particularly integrated learning systems, and outcomes with respect to literacy and numeracy. Auckland Uniservices, University of Auckland, 2000.

[9] Alabdulakareem, E., \& Jamjoom, M., Computer-assisted learning for improving ADHD individuals' executive functions through gamified interventions: A review. Entertainment Computing, 33, 2020, 100341.

[10] D. Rand, P. L. T. Weiss, N. Katz, Training multitasking in a virtual supermarket: A novel intervention after stroke. American Journal of Occupational Therapy, 63(5), 2009, 535-542.

[11] M. Jacoby, S. Averbuch, Y. Sacher, N. Katz, P. L. Weiss, R. Kizony, Effectiveness of executive functions training within a virtual supermarket for adults with traumatic brain injury: a pilot study. IEEE transactions on neural systems and rehabilitation engineering 21(2), 2012, 182-190.

[12] T. M. Fleming, L. Bavin, K. Stasiak, E. Hermansson-Webb, S. N. Merry, C. Cheek, ... S. Hetrick, Serious games and gamification for mental health: current status and promising directions. Frontiers in psychiatry, 7, 2017, 215.

[13] Dovis, S., Van der Oord, S., Wiers, R. W., \& Prins, P. J., Improving executive functioning in children with ADHD: Training multiple executive functions within the context of a computer game. A randomized double-blind placebo controlled trial. PloS one, 10(4), 2015, e0121651.

[14] Retalis, S., Korpa, T., Skaloumpakas, C., Boloudakis, M., Kourakli, M., Altanis, I., ... \& Pervanidou, P. (2014, October). Empowering children with ADHD learning disabilities with the Kinems Kinect learning games. In European Conference on Games Based Learning (Vol. 2, p. 469). Academic Conferences International Limited.

[15] P. M. Corsi, Human memory and the medial temporal region of the brain (Doctoral dissertation, ProQuest Information \& Learning), 1973.

[16] S. Della Sala, J. A. Foley, N. Beschin, M. Allerhand, R. H., Logie, Assessing dual-task performance using a paper-and-pencil test: Normative data. Archives of Clinical Neuropsychology, 25(5), 2010, 410-419.

[17] B.A. Eriksen, C.W. Eriksen, Effects of noise letters upon the identification of a target letter in a nonsearch task. Perception \& psychophysics, 16(1), 1974, 143-149.

[18] I. A. Chicchi Giglioli, C. de Juan Ripoll, E. Parra, M. A. Raya, EXPANSE: A novel narrative serious game for the behavioral assessment of cognitive abilities. PloS one, 13(11), 2018.

[19] I. A. Chicchi Giglioli, C. Bermejo Vidal, M. Alcañiz Raya, A virtual versus an augmented reality cooking task based-tools: a behavioral and physiological study on the assessment of executive functions. Frontiers in Psychology, 10, 2019, 2529. 